\title{
Neuronavigation in the Identification and Presurgical Planning of Cortical Focal Dysplasia
}

I

have read with great interest the article by Bartolini et $\mathrm{al}^{1}$ that appears in the previous issue of this journal.

The authors highlight the high sensitivity of 7T $\mathrm{T} 2{ }^{*}$-weighted MR imaging in the identification and anatomic delimitation of cortical focal dysplasia (CFD) type IIb, specifying that this study will help more precise surgical planning, as well as explain the cases of failure in epilepsy operations. While it is true that 7T MR imaging has these advantages, it is also true that not all centers where epilepsy surgery is performed have the possibility of having such a team.

Probably more affordable is the availability of a neuronavigation team that could achieve quite similar results. ${ }^{2}$

I present the case of a 3-year-old boy with a history of intellectual disability and 12-16 simple partial motor daily seizures secondarily generalized from 3 months of age despite different schemes of antiepileptic polytherapy, in which an extensive and diffuse right parieto-occipital cortico-subcortical lesion was diagnosed, congruent with the site of the onset of epileptogenic discharges.

With neuronavigator and neurophysiologic monitoring with a subdural grid electrode of 32 contacts placed over the lesion and 2 interhemispheric strip electrodes of 4 contacts, the anatomo-functional margins of the lesion could be identified with

http://dx.doi.org/10.3174/ajnr.A6417 high precision, and by means of a right parietal quadrantectomy with disconnection of the splenium, the entire lesion was resected (Figure).

Pathology confirmed CFD type IIb. At 18 months after the operation, the patient remained free of seizures (Engel I), with a sequential homonymous left hemianopia.

Neuronavigation proved to be an instrument of remarkable utility in the identification, delimitation, and complete resection of this voluminous cortical dysplasia. The advantage of the neuronavigation is that it allows a volumetric location of the lesion, which increases the accuracy of the resection of the lesion.

\section{REFERENCES}

1. Bartolini E, Cosottini M, Costagli M, et al. Ultra-high-field targeted imaging of focal cortical dysplasia: the intracortical black line sign in type IIb. AJNR Am J Neuroradiol 2019;40:2137-42 CrossRef Medline

2. Minkin K, Gabrovski K, Sirakov S, et al. Three-dimensional neuronavigation in SEEG-guided epilepsy surgery. Acta Neurochir (Wien) 2019;161:917-23 CrossRef Medline
(D) C.J. Valencia-Calderón Department of Neurosurgery Hospital Universitario Central de Asturias Oviedo, Spain 

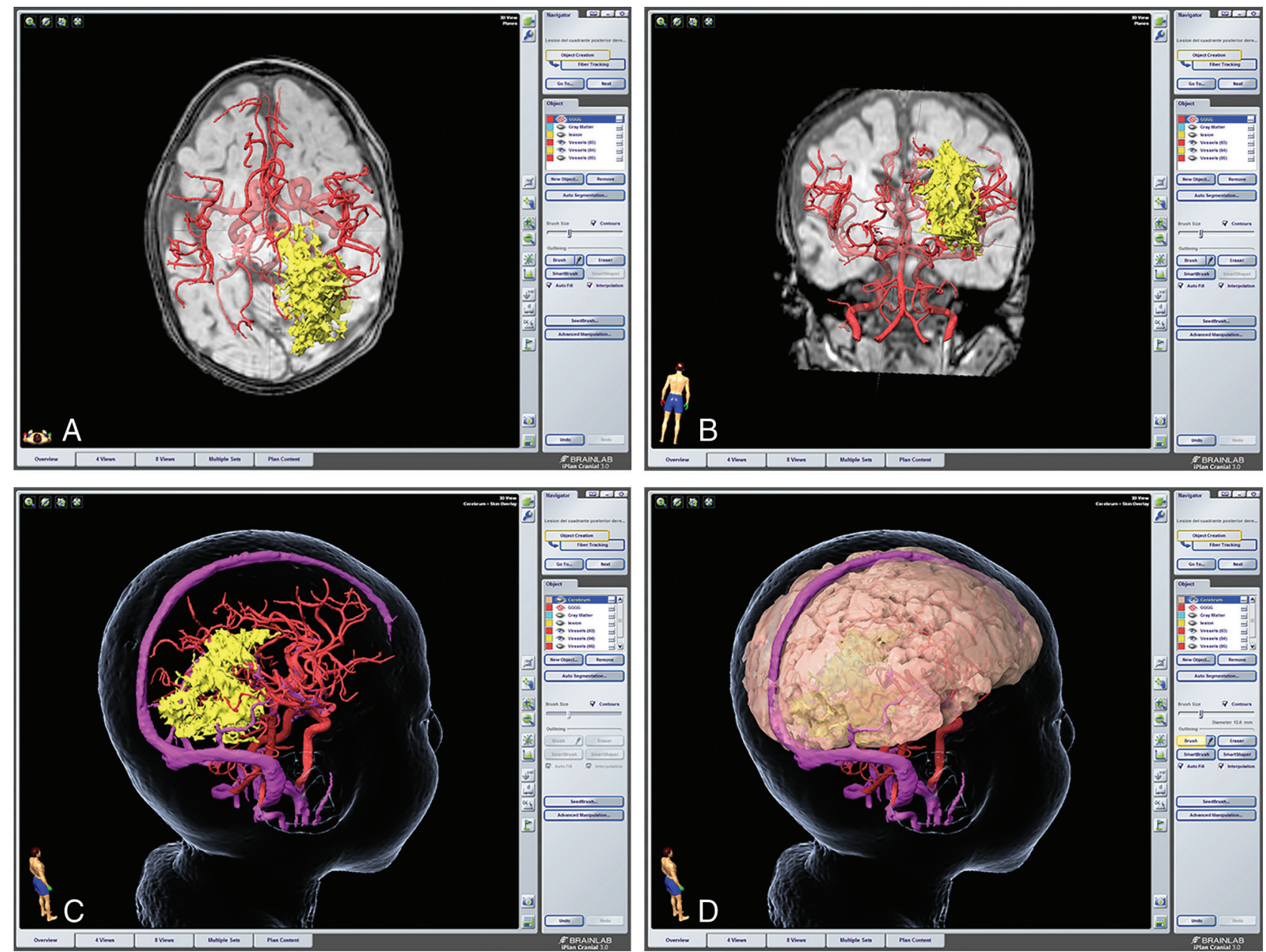

FIGURE. $A$ and $B$, Axial and coronal T2*-weighted MR imaging of a patient with long-standing epilepsy due to extensive cortical dysplasia. The right parieto-occipital cortical dysplasia has been rendered in yellow, and the circle of Willis, in red on the 3D model. $C$ and $D$, Transparent reconstruction of the patient's head with and without the brain where the circle of Willis and the venous sinus and vein of Labbé (in purple) are related to dysplasia. This image illustrates the surgical complexity of this dysplasia; planning the surgery took into consideration that endangering the vein of Labbé would carry the risk of venous infarction. Electrocorticography showed active spiking in the posterior cingulate gyrus, which has undergone complete resection. Final intraoperative electrocorticography showed no epileptiform activity. 